\title{
Editorial: Recent Advances in loT as a Service (IoTaas 2017)
}

\section{Der-Jiunn Deng ${ }^{1} \cdot$ Ai-Chun Pang ${ }^{2} \cdot$ Lajos Hanzo $^{3}$}

Published online: 29 April 2019

(C) Springer Science+Business Media, LLC, part of Springer Nature 2019

\section{Editorial:}

As we are entering the Internet of Things (IoT) era, a key question is how we can make the most of these compelling services for all stakeholders, including platform providers, IoT application developers, end-users, large and small organizations (such as city councils and enterprises) as well as manufacturers of smart devices. The number of smart devices supporting diverse facets of our daily lives spanning from manufacturing to clothing continues to grow, with an increasing thirst for more power, processing capability and network connectivity. The sheer size and variety of contextual data that they produce is enormous. It remains to be seen, how this huge potential will be turned into tangible utility; this special issue focuses on the discussions of the challenges posed by these trends.

The paradigm of "Everything as a Service" will ease the adoption of IoT based services and applications by end users, while forcing providers of smart objects and middleware platforms to build their solutions accordingly. To maximize impact and adoption, the barrier-to-entry should be lowered by making the development of new applications as well as the early adoption and exposure of smart objects as easy as possible. The goal of this special issue is to publish both state-ofthe-art and predictive papers on recent advances in "IoT as a Service" selected from the 3rd EAI International Conference on IoT as a Service (IoTaas 2017), which was held in Taichung (Taiwan) during September 20-22, 2017. After the event an

\section{Der-Jiunn Deng}

derjiunn.deng@gmail.com

Ai-Chun Pang

acpang@csie.ntu.edu.tw

Lajos Hanzo

lh@ecs.soton.ac.uk

1 National Changhua University of Education, No. 1, Jinde Road, Changhua City 500, Changhua County, Taiwan

2 National Taiwan University, No. 1, Section 4, Roosevelt Rd, Da'an District, Taipei City 10617, Taiwan

3 University of Southampton, University Road, Southampton SO17 1BJ, UK

open call was published to encourage the contributions presented at IoTaas 2017 to be extended and submitted to this special issue. After a rigorous review process, eleven high quality papers were selected for publication, which are briefly reviewed in the following.

These papers may be divided into two categories. Five papers are related to IoT applications, services and deployment. In the paper entitled "Flower Sermon: An Interactive Visual Design using IoTtalk" authored by Chung-Yun Hsiao, et al., Flower Sermon artwork is used as an example to show how interactive visual design can be conveniently implemented in IoTtalk, a platform conceived for IoT device interactions, which has been used to develop numerous IoT applications. The treatise "Home Healthcare Matching Service System Using the Internet of Things" by Tzong-Shyan Lin, et al. proposes a home healthcare matching service that lends patients the impression that the system is is indeed in charge of their healthcare, while all the licensing and other legal requirements, as well as the travel schedule restrictions of the healthcare professionals are met. The further contribution entitled "LaSa: Location Aware Wireless Security Access Control for IoT Systems", by Bingxian Lu, et al. proposes a crowdsourcing method for location aware security access control, namely LaSa, which is capable of confining wireless network access to certain physical areas by relying on a single commercial Access Point (AP). "A Survey of Localization Systems in Internet of Things", by Fekher Khelifi, et al. provides a general overview of the localization aspects of Wireless Sensor Networks (WSN) and surveys the pertinent technical details of localization techniques using relying on a diverse spectrum of technologies. Last, but not least, "A Hybrid Key Item Locating Method to Assist Elderly Daily Life Using Internet of Things", by Lun-Ping Hung, et al. proposes a beneficial hybrid calculation algorithm, which astutely combines the xBeacon sensing equipment, Received Signal Strength Indication (RSSI) based positioning, event analysis methods and an intelligent algorithm to construct a life-support mechanism for the home environment of senior citizens.

There are six papers in the second category, which address diverse connectivity, privacy and signal processing issues of the IoT. In the paper entitled "Interoperability in Internet of 
Things: Taxonomies and Open Challenges", by Mahda Noura, et al., a comprehensive survey on the state-of-the-art solutions for facilitating interoperability among different IoT platforms is performed. This survey categorized the existing proposals according to their interoperability handling techniques into the classes of gateways, virtual network and networking technologies, open APIs, SOA, semantic web technologies and open standards. The contribution entitled as "Evaluation of the Effect of Variations in Vehicle Velocity and Channel Bandwidth on an Image-Streaming System in Vehicular Networks", authored by Ming-Fong Tsai, et al. proposes a solution for implementing a real-time image-streaming system for vehicular networks. The treatise "The Digital Fingerprinting Analysis of Generic Twitter Sessions from Internet of Things Security Aspect", by Hai-Cheng Chu, et al. illustrates the 'digital bread crumbs' that the previous Twitter user left behind in diverse scenarios. Following the generic methodologies proposed, the digital forensics specialists operating either in the private sectors or the law enforcement agencies acting in the public sectors are capable of identifying the operations of the previous Twitter user via timeline analysis. The study entitled as "Collaboration IoT-based RBAC with Trust Evaluation Algorithm Model for Massive IoT Integrated Application", authored by Hsing-Chung Chen proposes the collaboration of IoT-aided Role-based Access Control combined with Trust Evaluation algorithms to reduce the probability of internal security threats both in intra-server and inter-server scenarios supporting large-scale integrated IoT applications. Finally, the special issue concludes with an article entitled "Loading Aware Green Power Control (LAGPC) for the Mitigation of Co-Tier Downlink Interference for Femtocell in the Future 5G Networks", authored by Kuo-Chang ting, et al. In this article, the authors propose a beneficial co-tier downlink interference reduction technique for densely deployed femto-cell base stations.

Acknowledgements As the Guest Editors of this special issue, we would like to thank all authors who have submitted papers to the special issue and in particular those whose papers have been accepted for this special issue after a rigorous peer-review. Assistance from the editorial staff of "Mobile Networks and Applications" is also much appreciated. Furthermore, the Guest Editors wish to acknowledge the kind assistance of all those valued Colleagues, who have generously dedicated their time to the review of papers submitted for potential inclusion in this special issue. Finally, our special thanks go to Dr. Imrich Chlamtac (editor-inchief) for his valuable support throughout the preparation of this special issue.

Publisher's Note Springer Nature remains neutral with regard to jurisdictional claims in published maps and institutional affiliations.

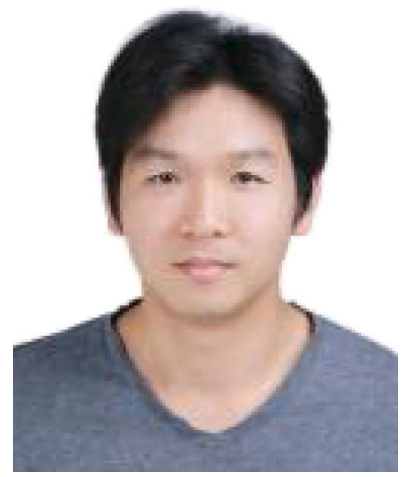

Der-Jiunn Deng (M'10) received the Ph.D. degree in electrical engineering from the National Taiwan University in 2005. He joined the National Changhua University of Education as an Assistant Professor in the Department of Computer Science and Information Engineering in August 2005 and then became a Distinguished Professor in August 2016. In July 2018, he was seconded to Overseas Chinese University as the Dean of research and development for a period of two years. Dr. Deng has received a number of research awards, such as the Research Excellency Award of National Changhua University of Education, the Outstanding Faculty Research Award of National Changhua University of Education, the ICS 2014 Best Paper award, the NCS 2017 Best Paper Award, and the Chinacom 2017 Best Paper Award. Dr. Deng is the Co-Editor-in-Chief of EAI Endorsed Transactions on IoT and Journal of Computers. He serves as an associate editor in IEEE Network Magazine and International Journal of Communication Systems. He also served or is serving on several program chairs, symposium chairs, and technical program committees for IEEE and other international conferences. His research interests include multimedia communication, quality-of-service, and wireless local network.

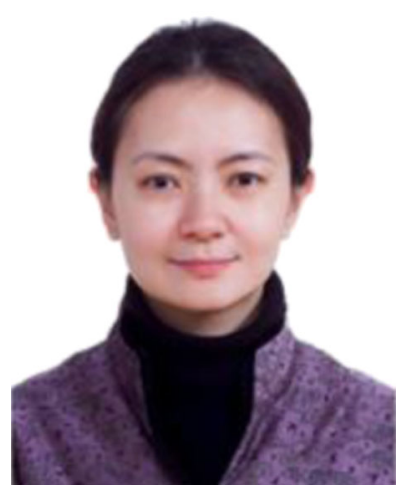

Ai-Chun Pang received the B.S., M.S. and Ph.D. degrees in Computer Science and Information Engineering from National Chiao Tung University, Taiwan, in 1996, 1998 and 2002, respectively. She joined the Department of Computer Science and Information Engineering (CSIE), National Taiwan University (NTU), Taipei, Taiwan in 2002. She is now a Professor in CSIE and INM, and is also an Adjunct Research Fellow of Research Center for Information Technology Innovation, Academia Sinica, Taiwan. Her research interests include the design and analysis of wireless and multimedia networking, mobile communications, and fog/edge computing. She is a senior member of the IEEE. 


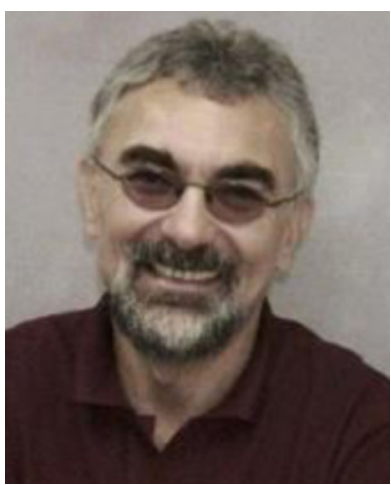

Lajos Hanzo FREng, FIEEE received his Masters degree in electronics in 1976 and his Doctorate in 1983 from the Technical University of Budapest. He holds two Honorary Doctorates, one from the Technical University of Budapest (2009) and one from the University of Edinburgh (2015). Since 1986 he has been with the University of Southampton, UK and in 2004 he was awarded the Doctor of Sciences (DSc) degree. Prior to that he has held research and academic posts in Hungary, Germany and the UK. Since 1998 he has been the head of the Communications Research Area at Southampton. He is also a former Chaired Professor at Tsinghua University, Beijing, China and a former EIC of the IEEE Press. Throughout his 42-year career he has coauthored 18 John Wiley - IEEE Press monographs and 1800+ research contributions at IEEE Xplore. 